\title{
Gallbladder adenomyomatosis: imaging findings, tricks and pitfalls
}

\author{
Matteo Bonatti ${ }^{1}$. Norberto Vezzali $^{1} \cdot$ Fabio Lombardo $^{1} \cdot$ Federica Ferro $^{1}$. \\ Giulia Zamboni $^{2}$ - Martina Tauber $^{3}$ - Giampietro Bonatti ${ }^{1}$
}

Received: 10 November 2016 / Revised: 13 January 2017 / Accepted: 17 January 2017 / Published online: 26 January 2017

(C) The Author(s) 2017. This article is published with open access at Springerlink.com

\begin{abstract}
Gallbladder adenomyomatosis (GA) is a benign alteration of the gallbladder wall that can be found in up to $9 \%$ of patients. GA is characterized by a gallbladder wall thickening containing small bile-filled cystic spaces (i.e., the Rokitansky-Aschoff sinuses, RAS). The bile contained in RAS may undergo a progressive concentration process leading to crystal precipitation and calcification development. A correct characterization of GA is fundamental in order to avoid unnecessary cholecystectomies. Ultrasound (US) is the imaging modality of choice for diagnosing GA; the use of high-frequency probes and a precise focal depth adjustment enable correct identification and characterization of GA in the majority of cases. Contrast-enhanced ultrasound (CEUS) can be performed if RAS cannot be clearly identified at baseline US: RAS appear avascular at CEUS, independently from their content. Magnetic resonance imaging (MRI) should be reserved for cases that are unclear on US and CEUS. At MRI, RAS can be identified with extremely high sensitivity, but their signal intensity varies widely according to their content. Positron
\end{abstract}

Based on the EPOS "Gallbladder adenomyomatosis: are we sure to know it?” DOI 10.1594/ecr2014/C-0198

Matteo Bonatti

matteobonatti@hotmail.com

1 Department of Radiology, Bolzano Central Hospital, 5 Boehler Street, 39100 Bolzano, Italy

2 Department of Radiology, University of Verona, 10 LA Scuro Place, 37134 Verona, Italy

3 Department of Pathology, Bolzano Central Hospital, 5 Boehler Street, 39100 Bolzano, Italy emission tomography (PET) may be helpful for excluding malignancy in selected cases. Computed tomography (CT) and cholangiography are not routinely indicated in the suspicion of GA.

\section{Teaching points}

1. Gallbladder adenomyomatosis is a common benign lesion (1-9\% of the patients).

2. Identification of Rokitansky-Aschoff sinuses is crucial for diagnosing gallbladder adenomyomatosis.

3. Sonography is the imaging modality of choice for diagnosing gallbladder adenomyomatosis.

4. Intravenous contrast material administration increases ultrasound accuracy in diagnosing gallbladder adenomyomatosis.

5. Magnetic resonance is a problem-solving technique for unclear cases.

Keywords Gallbladder - Rokitansky-Aschoff sinuses of the gallbladder · Gallbladder diseases · Ultrasonography · Magnetic resonance imaging

$\begin{array}{ll}\text { Abbreviations } \\ \text { GA } & \text { Gallbladder adenomyomatosis } \\ \text { RAS } & \text { Rokitansky-Aschoff sinuses } \\ \text { OC } & \text { Oral cholecystography } \\ \text { US } & \text { Trans-abdominal ultrasound } \\ \text { EUS } & \text { Endoscopic ultrasound } \\ \text { CEUS } & \text { Contrast-enhanced ultrasound } \\ \text { MRI } & \text { Magnetic resonance imaging } \\ \text { ADC } & \text { Apparent diffusion coefficient } \\ \text { CT } & \text { Computed tomography } \\ \text { PET } & \text { Positron emission tomography }\end{array}$




\section{Main text}

\section{Gallbladder adenomyomatosis}

Gallbladder adenomyomatosis (GA) is a benign alteration of the gallbladder wall characterized by excessive epithelial proliferation associated with hyperplasia of the muscularis propria, resulting in gallbladder wall thickening. The excessive epithelial proliferation leads to epithelial infolding within the underlying muscular layer with subsequent formation of epithelium-lined diverticular pouches, the so-called Rokitansky-Aschoff sinuses (RAS; Fig. 1) [1-4]. The content of RAS consists of bile that may undergo progressive dehydration over time, leading to cholesterine crystal precipitation [5]. Moreover, cholesterine crystals may induce a chronic inflammatory reaction leading to intramural dystrophic calcification development. The serosa is never involved by GA.

Adenomyomatosis may involve the gallbladder according to four main patterns: localized, segmental, annular and diffuse (Fig. 2) [6, 7].

Localized GA is the most common pattern and is characterized by a focal thickening, usually involving the fundal region (the so-called "fundal GA"). The uninvolved gallbladder wall appears physiologically thin and the overall gallbladder shape is usually maintained.

Segmental GA is characterized by the involvement of a larger portion of the gallbladder wall, typically the fundus and the distal third of the body. The involved portion appears contracted, whereas the uninvolved one maintains its normal shape.

Annular $G A$ is characterized by a ring-form thickening of the gallbladder wall, usually involving the middle portion. The gallbladder appears contracted only in the involved portion, changing its global morphology and becoming "hourglass-shaped". In some cases, epithelial proliferation may be particularly conspicuous and subdivide the gallbladder lumen into two separate compartments. As a consequence, biliary sludge and stones may accumulate into the isolated fundal compartment [8]. According to some authors, annular GA should be considered a subtype of segmental GA [9].

Diffuse GA is characterized by the involvement of the whole organ that consequently appears contracted, even after fasting.

The pathogenesis of GA is not fully understood: an association with gallbladder stones and chronic inflammatory changes has been highlighted in many studies [1, 5, 10-13], but a correlation with acquired wall motility as a consequence of increased endoluminal pressure has also been postulated $[14,15]$. GA is a benign lesion as the hyperplastic epithelium of GA has no higher neoplastic potential than that of a normal gallbladder, even though gallbladder carcinoma may also arise in association with GA [16]. Some studies have shown an increase in gallbladder cancer prevalence among patients with segmental type adenomyomatosis compared to patients without GA or with other patterns of GA, in particular, in the elderly. However, these results may have been influenced by the higher prevalence of cholecystolithiasis in patients

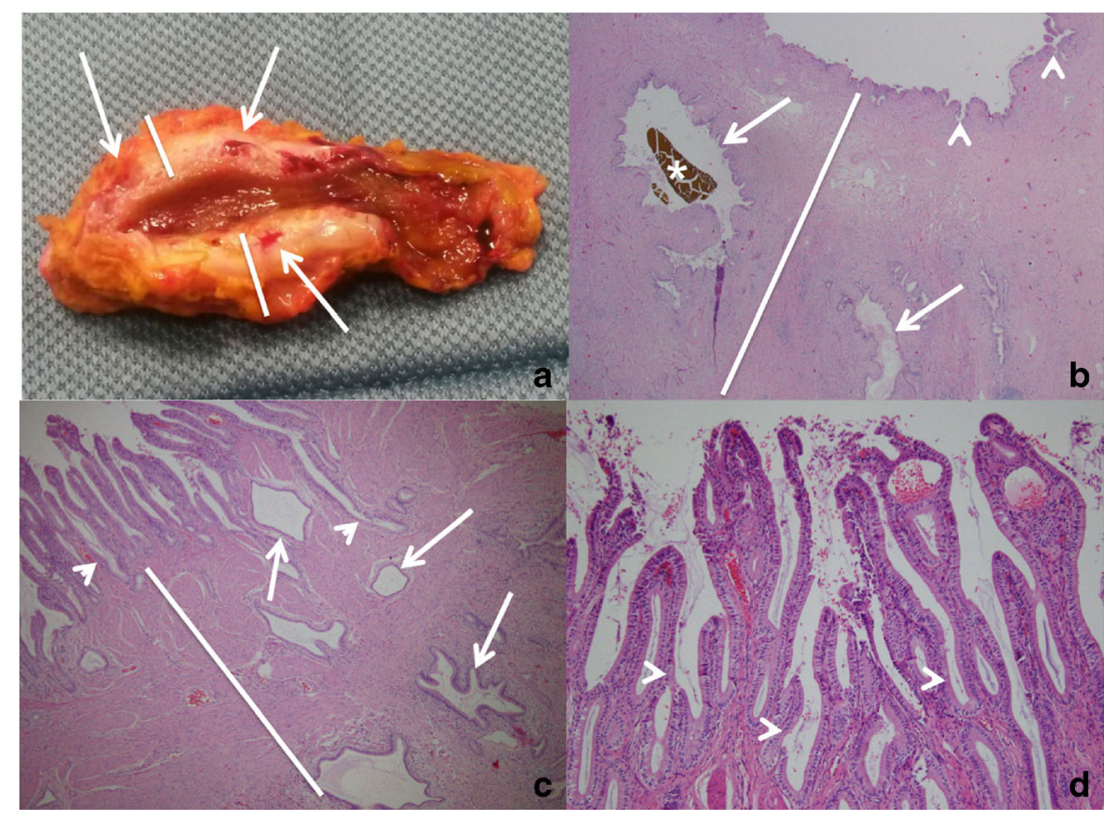

Fig. 1 Gallbladder adenomyomatosis: pathology findings. Macroscopically (a) GA is characterized by gallbladder wall thickening (lines) containing small cystic spaces (arrows) representing Rokitansky-Aschoff sinuses. Microscopically, at low $(2 \times)$ magnification (b and $\mathbf{c}$ ), wall thickening is due to hyperplasia of the muscular layer (lines); a variable degree of epithelial proliferation (arrowheads) is also appreciable and epithelium-lined cystic spaces, representing RAS (arrows), can be observed within the muscular layer. Biliary stones (star) may be present within RAS. At high $(40 \times)$ magnification $(\mathbf{d})$, the proliferative mucosal glandular component that leads to epithelial infolding (arrowheads) and RAS formation is better recognizable 

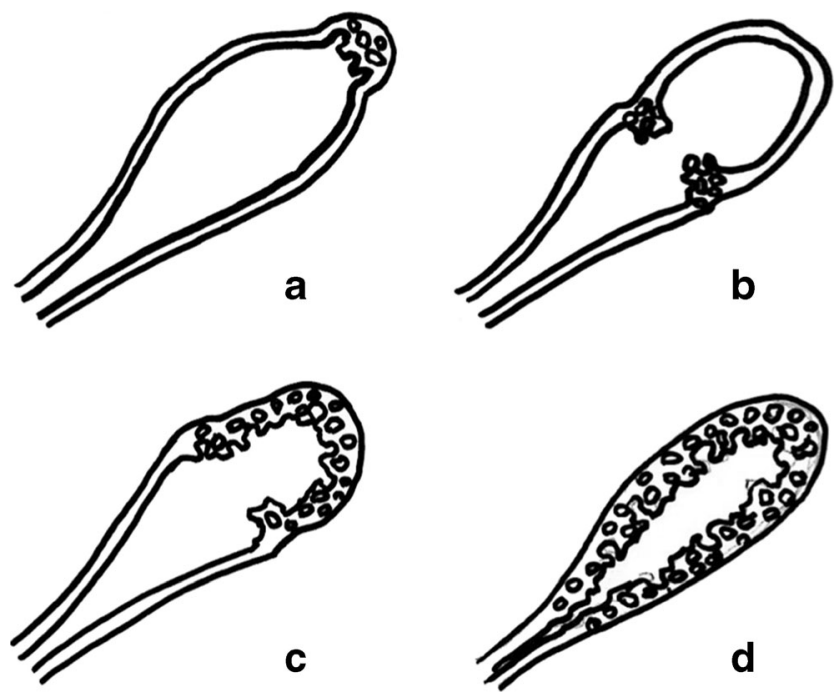

Fig. 2 Gallbladder adenomyomatosis: patterns of gallbladder wall involvement. Drawings showing localized gallbladder adenomyomatosis (a), annular gallbladder adenomyomatosis (b), segmental gallbladder adenomyomatosis (c) and diffuse gallbladder adenomyomatosis (d)

affected by segmental type GA, which represents a wellknown risk factor for gallbladder carcinoma [10-13, 16]. GA may increase in size over time and this change by itself must not be considered an index of malignancy [17]. Patients affected by GA are usually asymptomatic. When present, symptoms may include right upper quadrant pain, possibly also as a consequence of the presence of gallbladder stones.

GA is frequently observed in cholecystectomy specimens, with a reported prevalence of $1-9 \%$ in pathology series $[1,4,9,13]$. GA represents about $40 \%$ of benign gallbladder lesions $[1,4]$.

Given its relatively high prevalence and the continuous increase in imaging studies performance, GA can be frequently encountered during everyday practice. The radiologist plays a central role in the diagnosis of GA and its main aim is to distinguish GA from neoplastic gallbladder wall thickenings
(Fig. 3) in order to avoid unnecessary cholecystectomies. It is also important to accurately describe the gallbladder wall involvement pattern, as it can modify patient management.

There are no universally accepted guidelines for GA management. Given the lack of malignancy potential, GA is usually considered a "don't touch" lesion and cholecystectomy should be routinely reserved for symptomatic patients only or in case of inconclusive imaging findings. In any case, the surgical option might be considered in patients with segmental type GA, given its higher association with gallbladder cancer, and in patients with diffuse GA, given the possible difficulties in identifying neoplastic foci within the wall thickening [18].

In this article, we review multimodality imaging findings of GA, providing tips that may increase diagnostic confidence and highlighting possible pitfalls.

\section{Imaging of gallbladder adenomyomatosis}

Besides GA, differential diagnosis of gallbladder wall thickenings includes the post-prandial state, acute and chronic cholecystitis, cholesterine polyps, neoplasms and many other less common conditions. Independently from the radiological modality, an imaging clue for diagnosing GA is the detection, within a thickened gallbladder wall, of Rokitansky-Aschoff sinuses. It must be kept in mind that RAS may show extremely different imaging features according to their variable content that may range from clear bile to calcifications. Moreover, it must be considered that tiny cystic spaces, resembling RAS, have been identified also in rare cases of mucine-producing gallbladder carcinomas [19]; anyway, overall lesion shape in these neoplasms was much more irregular than in cases of GA.

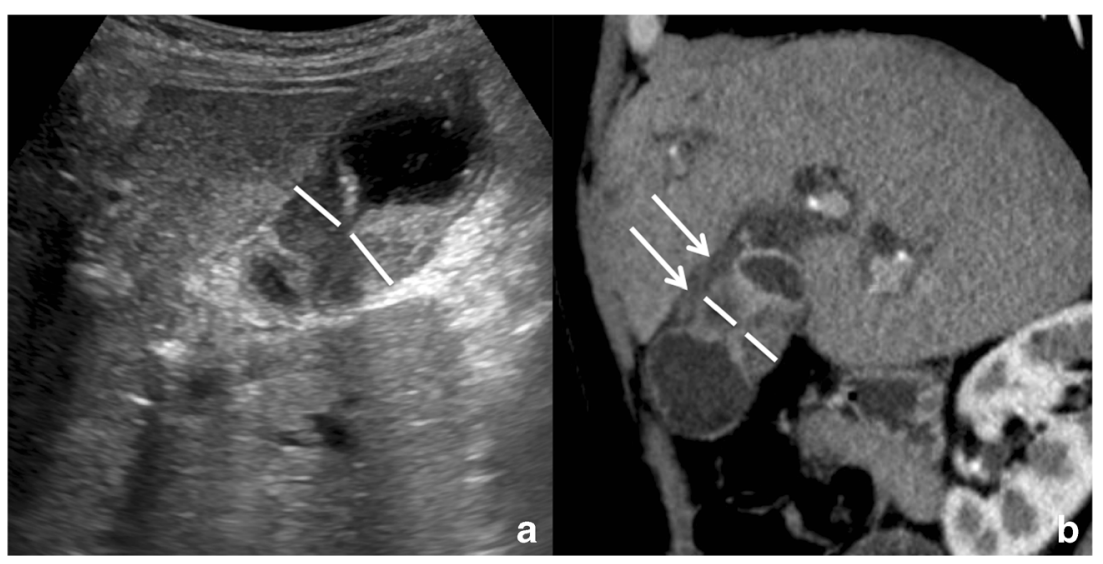

Fig. 3 Gallbladder cancer: gallbladder adenocarcinoma may involve the gallbladder wall with various patterns. This case of gallbladder adenocarcinoma with annular involvement (white lines) can be differentiated from an adenomyomatosis because of the absence of cystic spaces (RAS) within the wall thickening on ultrasound (a) and because of the presence of hypodense tissue between the gallbladder wall and the adjacent liver (arrows) on contrastenhanced CT (b) 


\section{Oral cholecystography}

Oral cholecystography (OC) was the first imaging modality used for diagnosing GA, but nowadays represents an obsolete technique. The knowledge of typical GA findings at OC, however, enables better understanding of the imaging patterns we are now dealing with. In particular, besides gallbladder wall thickening and possible strictures formation, the most relevant finding at $\mathrm{OC}$ was the visualization of rounded contrast media collections adjacent to the gallbladder lumen, representing RAS (Fig. 4) [20]. This finding represents the imaging demonstration of the communication between RAS and the gallbladder lumen.

\section{Ultrasound}

Trans-abdominal ultrasound (US) represents the imaging modality of choice for the detection and characterization of GA, with accuracy values that range from 91.5 to $94.8 \%$ in differentiating GA from early-stage gallbladder cancer [21].

\section{Imaging findings}

Focal or diffuse gallbladder wall thickening (Fig. 5) can be easily detected at US and represents the consequence of both epithelial hypertrophy and muscular hyperplasia. Wall thickening represents a hallmark of GA, being always present, but it is poorly specific, as it can be found in most gallbladder pathologies. Anyway, in GA, the outer gallbladder layer must appear sharp and a clear cleavage plane with the liver must always be present. No pericholecystic fluid should be observed.

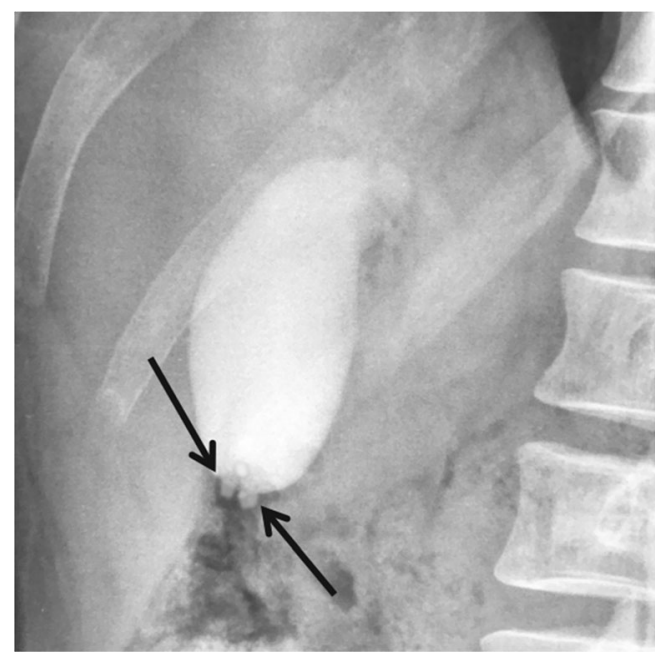

Fig. 4 Gallbladder adenomyomatosis: typical oral cholecystography findings. In this case of fundal type GA, RAS (arrows) are filled by contrast material as a consequence of their communication with the gallbladder lumen. Courtesy of Marco Ferigato, radiographer at Bolzano Central Hospital
Small anechoic cystic spaces $(1-10 \mathrm{~mm})$ representing clear bile-filled RAS should be recognized within the thickened gallbladder, being pathognomonic for GA (Fig. 5a). Whenever cholesterine crystals fill RAS, they appear as intramural echogenic spots in association with reverberation artefacts (Fig. $5 b$ and e). Reverberation artefacts are the consequence of the coexistence of different acoustic impedance media, i.e., clear bile and cholesterine crystals, within RAS and appear as hyperechoic "comet-tail" artefacts that project deeply into RAS. Sometimes RAS themselves may be not directly recognizable at the origin of reverberation artefacts. Calcification-filled RAS appear as intramural echogenic spots associated with posterior acoustic shadowing (Fig. 5c). Also, the presence of cholesterine crystal- or calcification-filled RAS is virtually diagnostic for GA.

Twinkling artefacts on colour Doppler ultrasound (Fig. 5d) are due to the interaction of the ultrasound beam with a rough acoustic interface composed by randomly disposed strongly reflecting media (i.e., cholesterine crystals or calcifications) [22]. Twinkling artefacts appear as rapidly alternating red and blue colour Doppler signals, "comet-tail" shaped, deeply in RAS, and are better appreciable using low-frequency probes [23]. Their presence is strongly associated with GA.

\section{Tips \& tricks}

Patient's fasting is fundamental in order to correctly evaluate the gallbladder and, in particular, whenever dealing with gallbladder wall thickenings. A minimum of $8 \mathrm{~h}$ of fasting is recommended before upper abdomen sonography.

A precise focal depth adjustment is crucial in order to correctly investigate every portion of the gallbladder wall for the presence of GA. In particular, it is often necessary to set the focal point to a very superficial position in order to evaluate patients with fundal type GA.

The use of high-frequency probes (Fig. 6) increases US accuracy in the diagnosis of GA. Indeed, GA often involves gallbladder fundus, which is usually unsatisfactorily evaluated by means of the classical $4-5-\mathrm{MHz}$ convex probes; every suspicious finding in this area must be further investigated by means of higher frequency $(7-9 \mathrm{MHz})$ linear probes for better characterization.

The introduction of harmonic imaging has increased US accuracy in depiction of gallbladder wall morphology and in detection of Rokitansky-Aschoff sinuses. Harmonic imaging should always be used in the suspicion of GA.

GA is sometimes poorly visible with the classical subcostal approach, particularly in obese patients. The interposition of hepatic parenchyma between the probe and the gallbladder wall (i.e., the so-called hepatic window) may overcome this limitation, increasing image quality. 
Fig. 5 Gallbladder adenomyomatosis: typical US findings in annular type (a), fundal type (b and d), segmental type (c) and diffuse type (e).

Gallbladder wall thickening (line) is always seen in gallbladder adenomyomatosis, but it is nonspecific. On b-mode images, Rokitansky-Aschoff sinuses (arrows) typically appear anechoic (a), but they can also appear hyperechoic if cholesterine crystals or calcifications are present (b and $\mathbf{c})$. Comet-tail reverberation artefacts (Figures $b$ and $e$, arrowheads) or acoustic shadowing (c, arrowheads) are usually observed profoundly in RAS. On colour Doppler images (d), twinkling artefacts (arrowheads) may be observed profoundly in RAS
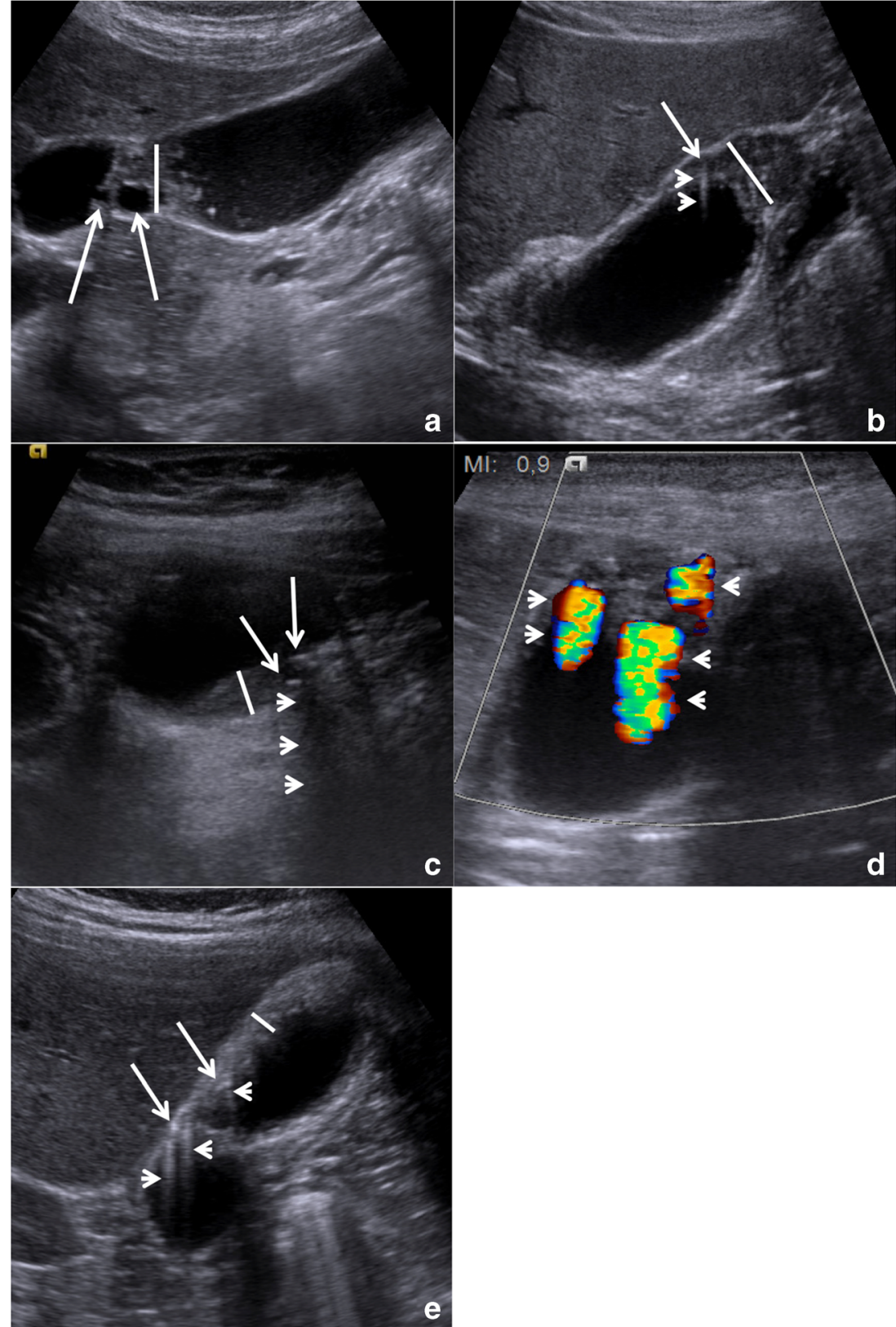

\section{Pitfalls \& limitations}

US is an operator-dependent imaging modality and the ability in depicting GA varies according to the operator's experience. Moreover, sonographic examination may be limited in case of obese patients and bowel gas interposition.

A possible imaging pitfall is the differentiation between cholesterine polyps and GA. Polyps appear as solid nodules with exophytic growth inside the gallbladder lumen, whereas GA appears as focal or diffuse mural thickening. Anyway, polyps and adenomyomatosis may coexist in some patients (Fig. 7).

Large round hyperechoic intramural collections without acoustic shadowing or reverberation artefacts, representing cholesterine-filled RAS (Fig. 8), may sometimes be observed. This finding may cause diagnostic doubts.

\section{Endoscopic ultrasound}

Endoscopic ultrasound (EUS) is an invasive imaging modality that is capable of accurately evaluating the gallbladder wall as the high-frequency probe can be positioned in its close proximity without the interposition of other anatomical structures. This results in a higher accuracy in the evaluation of gallbladder wall thickenings in comparison to US. EUS findings are the same as trans-abdominal ones (i.e., gallbladder wall thickening with intramural cystic 


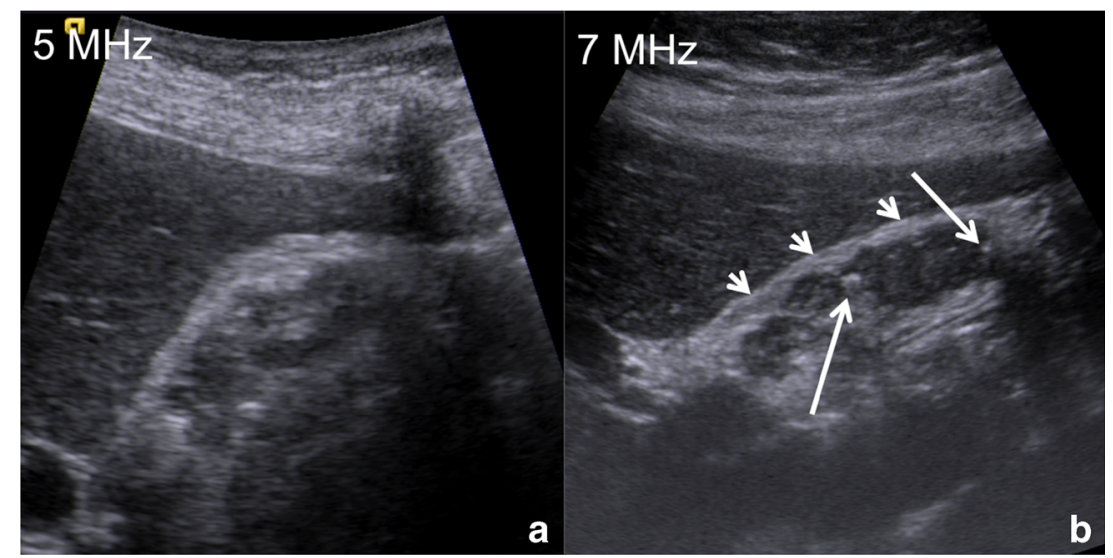

Fig. 6 US of gallbladder adenomyomatosis: use of different frequencies probes. In this patient with diffuse GA, the gallbladder wall is poorly evaluable by means of a conventional $5-\mathrm{MHz}$ convex probe (a). Using a high-resolution 7-MHz linear probe (b) hyperechoic Rokitansky-

spaces and/or echogenic foci, comet-tail artefacts and twinkling artefacts) and can be highlighted with higher sensitivity, in particular, in obese patients [24]. The main limitations to EUS reside in its invasiveness, low tolerability and costs; therefore, EUS is not routinely considered for the diagnosis of GA. Moreover, it has been demonstrated that EUS can depict some microcystic spaces that may be present in gallbladder cancer [8].

\section{Contrast-enhanced ultrasound}

Intravenous administration of micro-bubble contrast material represents a useful complement to conventional US and is

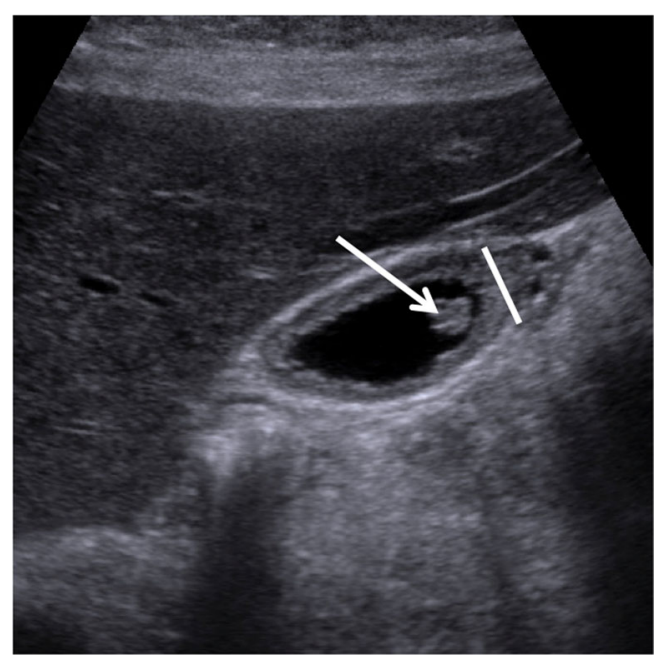

Fig. 7 US of gallbladder adenomyomatosis: differential diagnosis with cholesterine polyps. Cholesterine polyps (arrow) must not be confused with gallbladder adenomyomatosis (line); however, the two alterations may coexist in the same patient
Aschoff sinuses (arrows) can be highlighted within a diffusely thickened gallbladder wall; moreover, the serosa maintains sharp margins (arrowheads)

increasingly used for various indications in abdominal imaging (e.g., for the differential diagnosis of focal liver lesions and for the characterization of renal cysts). In recent years, contrast enhanced ultrasound (CEUS) has been proposed, with encouraging results, also for the differential diagnosis of gallbladder wall thickenings $[25,26]$ and Tang et al. have demonstrated that contrast material administration significantly increases US sensitivity in the detection of RAS and in the depiction of gallbladder wall continuity in patients with GA [27]. CEUS implicates the use of dedicated low mechanical index presets and intravenous administration of a bolus of $2.4 \mathrm{ml}$ of contrast material, containing $8 \mu \mathrm{l} / \mathrm{ml}$ sulphur hexafluoride microbubbles, followed by a $10-\mathrm{ml}$ saline flush; the target lesion is then scanned for the following 3-5 min in order to assess its vascularization.

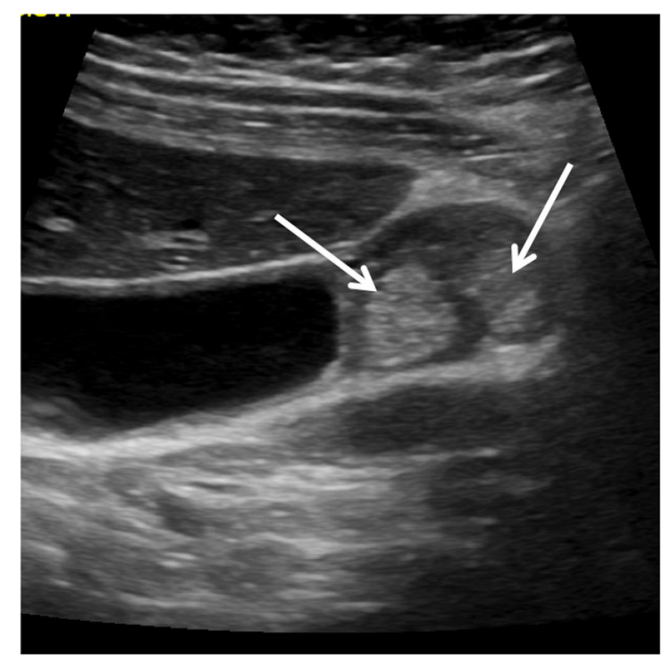

Fig. 8 US of gallbladder adenomyomatosis: pitfalls. Cholesterine crystals may accumulate within large Rokitansky-Aschoff sinuses, determining a hyperechoic aspect (arrows) without acoustic shadowing 


\section{Imaging findings}

The thickened gallbladder wall shows the same degree of enhancement as the adjacent normal wall in the majority of the cases, whereas a relative hyper-enhancement may be observed in about $15 \%$ of the cases. Wall enhancement typically shows a trilaminar pattern during the arterial phase as a consequence of increased mucosal and serosal vascularization. The external layer must show no discontinuities.

Avascular spaces, representing $R A S$, must be observed within the thickened gallbladder wall (Fig. 9). RAS appear avascular in every phase of the dynamic study, independently from their content. The identification of avascular spaces within a gallbladder wall thickening is virtually pathognomonic for GA.

\section{Tips \& tricks}

Intramural avascular spaces are best appreciated 70-100 $s$ after endovenous contrast material administration when the gallbladder wall shows a homogeneous high-degree enhancement.

\section{Pitfalls \& limitations}

The low mechanical index preset used for the performance of CEUS may reduce the accuracy in the characterization of gallbladder wall thickenings located in deep positions, away from the abdominal surface and from the probe. This may significantly limit CEUS accuracy in lesions involving the gallbladder neck and in obese patients.

\section{Magnetic resonance}

Thanks to the recent technical developments, to its multiplanarity and to its high tissue contrast resolution, magnetic resonance imaging (MRI) is becoming an increasingly requested imaging technique for the study of gallbladder pathologies [9]. On the other hand, MRI remains a time- and resource-consuming imaging modality and, therefore, the indications for its use must be accurately evaluated. MRI is the imaging modality that offers the highest accuracy in diagnosing GA and, in particular, in differentiating GA from gallbladder carcinoma (accuracy 93.0\%) [28].

\section{Imaging findings}

Gallbladder wall thickening can be clearly depicted both on T1- and on T2-weighted images, and is not a specific finding. Anyway, MRI warrants high specificity in the diagnosis of GA by accurately ruling out extra-parietal infiltration, which is indicative of gallbladder carcinoma.

RAS typically appear markedly hyperintense on T2weighted images (Fig. 10) [29], hypointense on T1-weighted images and show no contrast enhancement. Anyway, progressive bile concentration and calcification development may change the MRI appearance of RAS that may become increasingly hyperintense on T1-weighted images (Fig. 11) and relatively hypointense on T2-weighted ones.

\section{Tips \& tricks}

The use of fat-saturated T2-weighted sequences increases RAS conspicuity.

MR cholangiopancreatography (MRCP) images can be useful for identifying RAS that sometimes may be overlooked on axial images. On MRCP images, many RAS can be usually observed one next to each other along the involved gallbladder wall leading to the so-called pearl necklace sign (Fig. 12) [30].

RAS may be extremely small and, therefore, barely recognizable on thick slab T2-weighted images. The acquisition of volumetric respiratory-triggered $\mathrm{T} 2$-weighted images increases the sensitivity of MRI in recognizing small RAS and offers the possibility of multiplanar reconstructions.

Contrast material administration is not routinely indicated in the suspicion of GA. Indeed, heavily T2-weighted fast spin-
Fig. 9 Gallbladder

adenomyomatosis: typical contrast-enhanced ultrasound (CEUS) findings. On CEUS, the thickened gallbladder wall shows discrete contrast enhancement, whereas Rokitansky-Aschoff sinuses (arrows) appear as avascular structures during every phase of the exam

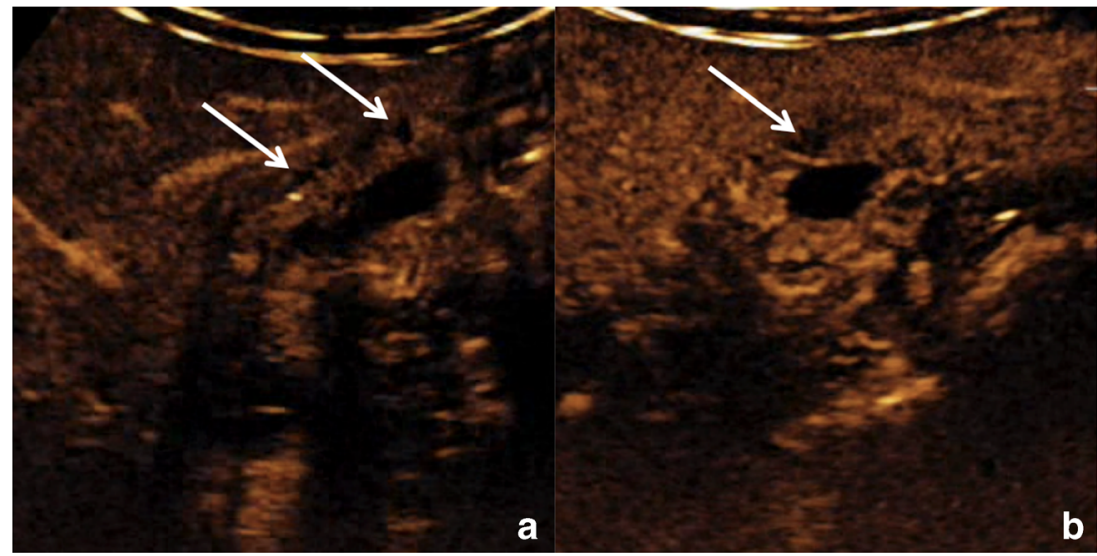


Fig. 10 Gallbladder

adenomyomatosis: typical MRI

findings. On MRI, GA can be identified as a mural thickening (line) containing small T2-

hyperintense spaces representing RAS (arrows). RAS can be better identified on fat-saturated T2weighted images (b) than on nonfat-saturated ones (a)

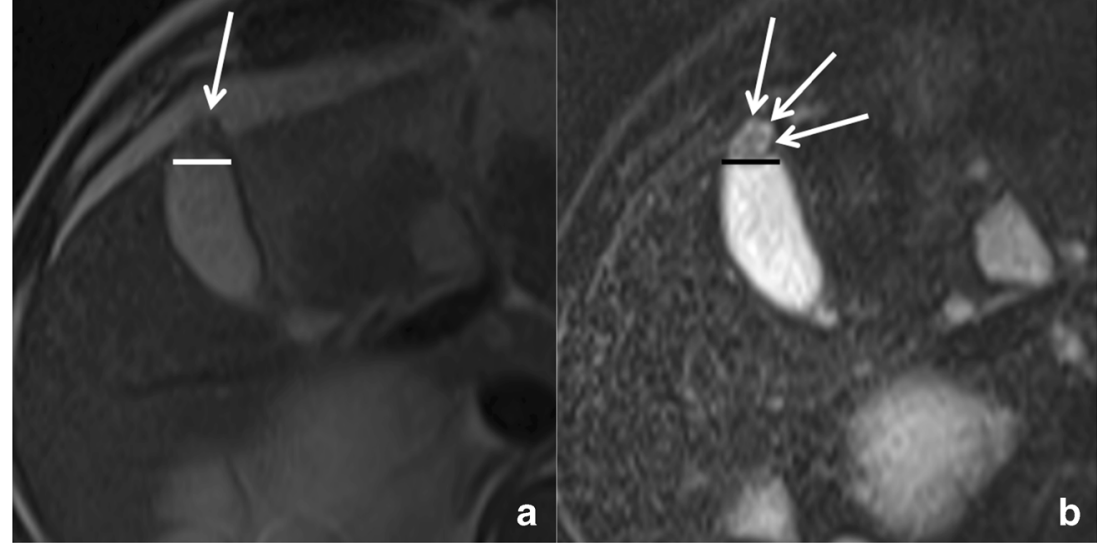

echo sequences are the most reliable for the identification of RAS.

Diffusion-weighted imaging can be helpful in the differential diagnosis between benign and malignant gallbladder wall thickenings. Ogawa et al. [31] demonstrated that malignant thickenings show significantly lower apparent diffusion coefficient (ADC) values than benign ones. Despite this, some overlap exists between benign and malignant lesions.

\section{Pitfalls \& limitations}

T1-hyperintense RAS can be frequently observed. This must not raise any doubt in the diagnosis of GA, being the consequence of concentrated biliary content, cholesterine crystals or calcifications.

RAS with different content, and consequently with different signal intensities, often coexist in the same patient.

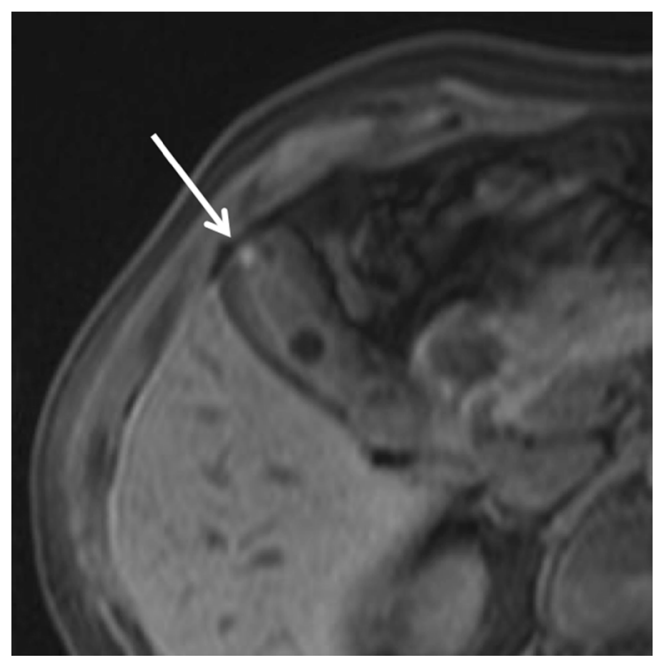

Fig. 11 MRI of gallbladder adenomyomatosis: T1-hyperintense Rokitansky-Aschoff sinuses. Rokitansky-Aschoff sinuses (arrow) may appear hyperintense on T1-weighted images if containing concentrated bile or calcifications

\section{Computed tomography}

The finding of a gallbladder wall thickening at computed tomography (CT) may represent a diagnostic dilem$\mathrm{ma}$; in fact, unless clear signs of malignancy are present, CT has an unsatisfactory accuracy in their differential diagnosis. The accuracy of $\mathrm{CT}$ in differentiating GA from gallbladder carcinoma is between 40 and $75 \%$ [32] and a confident diagnosis of GA is possible only if large (at least 3-4 mm) RAS are present.

\section{Imaging findings}

Gallbladder wall thickening without extra-parietal growth can be confidently observed at $\mathrm{CT}$, with sensitivity comparable to the other modalities.

Well-delimitated hypodense intramural spaces, representing RAS, can be confidently recognized only if they

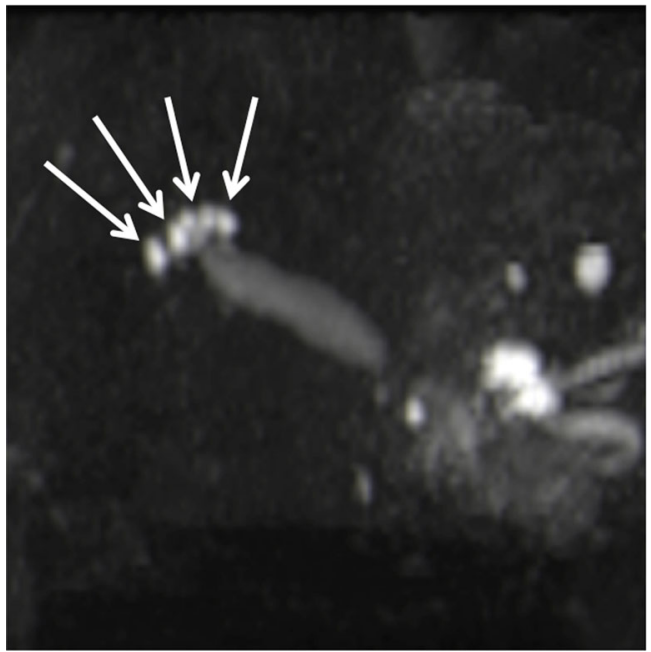

Fig. 12 MRI of gallbladder adenomyomatosis: the pearl necklace sign. On heavily T2-weighted images, like in this maximum intensity projection reconstruction of a volumetric MRCP, a lot of RAS can be identified one next to the other around the gallbladder, leading to the so-called pearl necklace sign 
Fig. 13 Gallbladder adenomyomatosis: typical CT findings. At CT, gallbladder adenomyomatosis is characterized by mural thickening representing Rokitansky-Aschoff sinuses (arrows). Large RAS can be easily identified on 3-mmthick reconstructions (a), whereas for identifying smaller RAS thin slices evaluation is crucial (b) (line) containing cystic spaces

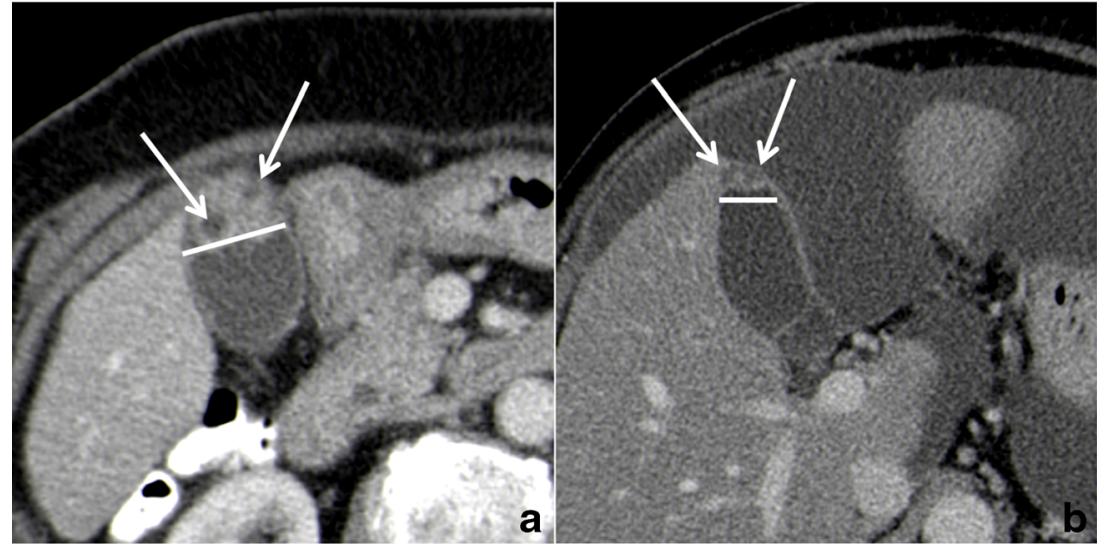

Fig. 14 CT of gallbladder adenomyomatosis: intramural calcifications. CT accurately depicts intramural calcifications (arrows) that may develop within Rokitansky-Aschoff sinuses and which are pathognomonic for gallbladder adenomyomatosis

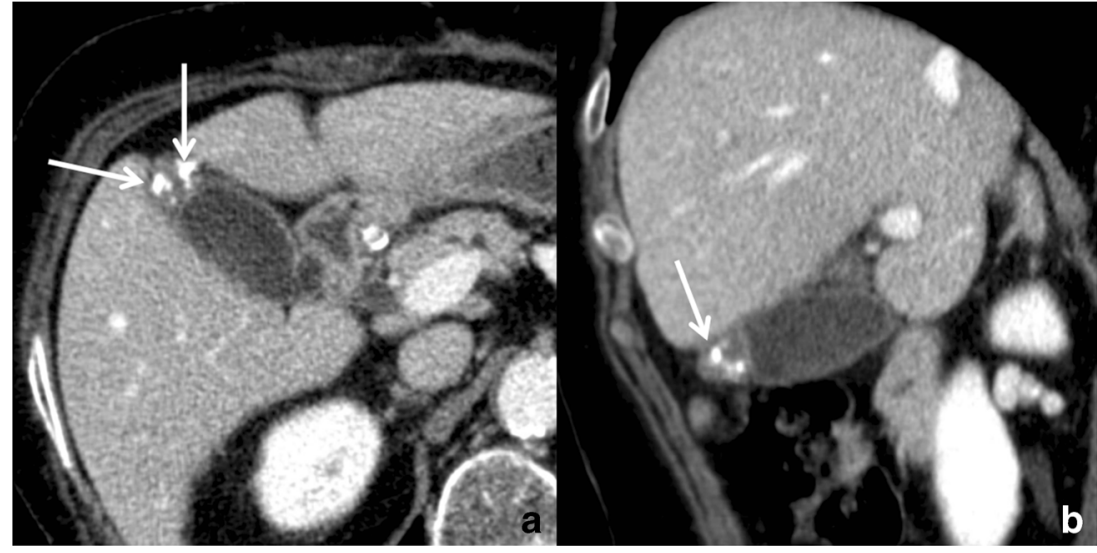

reach 3-4 $\mathrm{mm}$ in diameter and if they have a clear bile content (Fig. 13). If RAS are clearly identified, CT diagnosis of GA can be made.

Focal intramural calcifications (Fig. 14) are virtually pathognomonic for GA. Unfortunately, GA shows intramural calcifications only in a minority of the cases.

\section{Tips \& tricks}

CT images evaluation using thin slice thickness (1-2 mm) increases accuracy in identifying RAS and in excluding extramural infiltration, although it shows more background noise compared to thicker reconstructions.

Endovenous contrast material administration is fundamental in order to increase $\mathrm{CT}$ accuracy in RAS recognition and to exclude the presence of extra-parietal growth.

\section{Pitfalls \& limitations}

Due to their tiny dimensions and to the low tissue contrast resolution of the technique, RAS cannot be clearly identified at $\mathrm{CT}$ in the majority of the cases.
Fig. 15 Gallbladder

adenomyomatosis: typical PET-

CT findings. Gallbladder

adenomyomatosis (circle) usually shows an F-18FDG uptake equal or lower than the adjacent liver
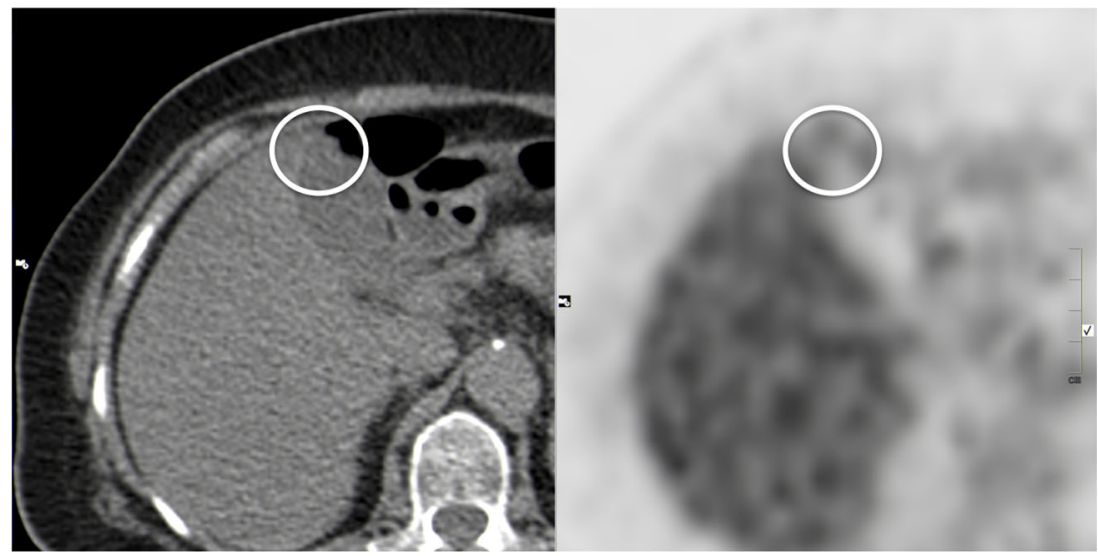


\section{Positron emission tomography}

Despite its low spatial resolution, positron emission tomography (PET) offers the possibility of obtaining metabolic information from body tissues. After i.v. administration of fluorine18-fluorodeoxiglucose (18 F-FDG), high glucose-consuming tissues (i.e., neoplastic and inflammatory ones) can be identified. PET is not usually performed in the suspicion of GA, but patients affected by GA may sometimes undergo PET for other reasons.

\section{Imaging findings}

GA typically shows no 18 F-FDG uptake or lower uptake compared to the liver (Fig. 15). This finding is not specific for GA, but may help in excluding malignancy [33].

\section{Pitfalls \& limitations}

An acute inflammatory reaction sometimes surrounds RAS, generating an increased 18 F-FDG uptake that leads to false positive diagnosis of neoplasm [34].

PET has low spatial resolution and its accuracy in excluding early gallbladder neoplasms may be unsatisfactory in lesions measuring less then $1 \mathrm{~cm}$.

\section{Conclusions}

US represents the imaging modality of choice for diagnosing GA, whereas CEUS should be considered the second-line imaging modality. MRI is the problem-solving technique for unclear cases at US and CEUS. PET may be considered for excluding malignancy in cases undiagnosed at US, CEUS and MRI. CT, endoscopic ultrasound and oral cholecystography are not routinely considered for diagnosing GA.

Open Access This article is distributed under the terms of the Creative Commons Attribution 4.0 International License (http:// creativecommons.org/licenses/by/4.0/), which permits unrestricted use, distribution, and reproduction in any medium, provided you give appropriate credit to the original author(s) and the source, provide a link to the Creative Commons license, and indicate if changes were made.

\section{References}

1. Jutras JA (1960) Hyperplastic cholecystoses; hickey lecture, 1960. Am J Roentgenol Radium Therapy, Nucl Med 83:795-827

2. Kai K, Ide T, Masuda $M$ et al (2011) Clinicopathologic features of advanced gallbladder cancer associated with adenomyomatosis. Virchows Arch 459:573-580

3. Bevan G (1970) Acalculous adenomyomatosis of the gallbladder. Gut 11:1029-1034
4. Colquhoun J (1961) Adenomyomatosis of the gall-bladder (intramural diverticulosis). Br J Radiol 34:101-112

5. Cariati A, Cetta F (2003) Rokitansky-aschoff sinuses of the gallbladder are associated with black pigment gallstone formation: a scanning electron microscopy study. Ultrastruct Pathol 27:265-270

6. Aguirre JR, Bohler RO, Guraieb S (1969) Hyperplastic cholecystoses; a new contribution to the Unitarian theory. Am J Roentgenol Radium Therapy, Nucl Med 107:1-13

7. Bonatti M, Ferro F, Gentile L, Pozzi Mucelli R, Bonatti G (2014) Gallbladder Adenomyomatosis: Are We Sure to Know It? EPOS. doi:10.1594/ecr2014/c-0198

8. Hammad AY, Miura JT, Turaga KK, Johnston FM, Hohenwalter MD, Gamblin TC (2016) A literature review of radiological findings to guide the diagnosis of gallbladder adenomyomatosis. HPB 18:129-135

9. Catalano OA, Sahani DV, Kalva SP et al (2008) MR imaging of the gallbladder: a pictorial essay. Radiographics 28:135-155, quiz 324

10. Kai K (2016) Organ-specific concept and controversy for premalignant lesions and carcinogenesis of gallbladder cancer. Hepatobiliary Surg Nutr 5:85-87

11. Nabatame N, Shirai Y, Nishimura A, Yokoyama N, Wakai T, Hatakeyama K (2004) High risk of gallbladder carcinoma in elderly patients with segmental adenomyomatosis of the gallbladder. J Exp Clin Cancer Res 23:593-598

12. Nishimura A, Shirai Y, Hatakeyama K (2004) Segmental adenomyomatosis of the gallbladder predisposes to cholecystolithiasis. J Hepato-Biliary-Pancreat Surg 11:342-347

13. Ootani T, Shirai Y, Tsukada K, Muto T (1992) Relationship between gallbladder carcinoma and the segmental type of adenomyomatosis of the gallbladder. Cancer 69:2647-2652

14. Kim JH, Jeong IH, Han JH et al (2010) Clinical/pathological analysis of gallbladder adenomyomatosis; type and pathogenesis. Hepato-Gastroenterology 57:420-425

15. Williams I, Slavin G, Cox A, Simpson P, de Lacey G (1986) Diverticular disease (adenomyomatosis) of the gallbladder: a radiological-pathological survey. Br J Radiol 59:29-34

16. Yoon JH, Cha SS, Han SS, Lee SJ, Kang MS (2006) Gallbladder adenomyomatosis: imaging findings. Abdom Imaging 31:555-563

17. Agrawal S, Khurana J, Daruwala C (2012) Gallbladder adenomyomatosis: a malignant masquerader. Dig Liver Dis 44:e23

18. Pellino G, Sciaudone G, Candilio G et al (2013) Stepwise approach and surgery for gallbladder adenomyomatosis: a mini-review. Hepatobiliary Pancreat Dis Int 12:136-142

19. Yoshimitsu K, Irie H, Aibe $\mathrm{H}$ et al (2005) Well-differentiated adenocarcinoma of the gallbladder with intratumoral cystic components due to abundant mucin production: a mimicker of adenomyomatosis. Eur Radiol 15:229-233

20. March HC (1948) Visualization of the rokitansky-aschoff sinuses of the gallbladder during cholecystography. Am J Roentgenol Radium Ther 59:197-203

21. Joo I, Lee JY, Kim JH et al (2013) Differentiation of adenomyomatosis of the gallbladder from early-stage, wallthickening-type gallbladder cancer using high-resolution ultrasound. Eur Radiol 23:730-738

22. Kim HC, Yang DM, Jin W, Ryu JK, Shin HC (2010) Color Doppler twinkling artifacts in various conditions during abdominal and pelvic sonography. J Ultrasound Med 29:621-632

23. Yu MH, Lee JY, Yoon JH, Baek JH, Han JK, Choi BI (2012) Color Doppler twinkling artifacts from gallbladder adenomyomatosis with $1.8 \mathrm{MHz}$ and $4.0 \mathrm{MHz}$ color Doppler frequencies. Ultrasound Med Biol 38:1188-1194

24. Kim HJ, Park JH, Park DI et al (2012) Clinical usefulness of endoscopic ultrasonography in the differential diagnosis of gallbladder wall thickening. Dig Dis Sci 57:508-515

25. Sparchez Z, Radu P (2012) Role of CEUS in the diagnosis of gallbladder disease. Med Ultrason 14:326-330 
26. Xie XH, Xu HX, Xie XY et al (2010) Differential diagnosis between benign and malignant gallbladder diseases with real-time contrast-enhanced ultrasound. Eur Radiol 20:239-248

27. Tang S, Huang L, Wang Y, Wang Y (2015) Contrast-enhanced ultrasonography diagnosis of fundal localized type of gallbladder adenomyomatosis. BMC Gastroenterol 15:99

28. Yoshimitsu K, Honda H, Aibe $\mathrm{H}$ et al (2001) Radiologic diagnosis of adenomyomatosis of the gallbladder: comparative study among MRI, helical CT, and transabdominal US. J Comput Assist Tomogr 25:843-850

29. Yoshimitsu K, Honda H, Jimi M et al (1999) MR diagnosis of adenomyomatosis of the gallbladder and differentiation from gallbladder carcinoma: importance of showing rokitansky-aschoff sinuses. AJR Am J Roentgenol 172:1535-1540

30. Haradome H, Ichikawa T, Sou H et al (2003) The pearl necklace sign: an imaging sign of adenomyomatosis of the gallbladder at MR cholangiopancreatography. Radiology 227:80-88
31. Ogawa T, Horaguchi J, Fujita N et al (2012) High b-value diffusionweighted magnetic resonance imaging for gallbladder lesions: differentiation between benignity and malignancy. J Gastroenterol 47: $1352-1360$

32. Ching BH, Yeh BM, Westphalen AC, Joe BN, Qayyum A, Coakley FV (2007) CT differentiation of adenomyomatosis and gallbladder cancer. AJR Am J Roentgenol 189:62-66

33. Suzuki K, Watada S, Yoko M, Nakahara T, Kumamoto Y (2011) Successful diagnosis of gallbladder carcinoma coexisting with adenomyomatosis by $18 \mathrm{~F}-\mathrm{FDG}-\mathrm{PET}$-report of a case. J Gastrointest Cancer 42:252-256

34. Maldjian PD, Ghesani N, Ahmed S, Liu Y (2007) Adenomyomatosis of the gallbladder: another cause for a "hot" gallbladder on 18F-FDG PET. AJR Am J Roentgenol 189:W36-38 